\title{
ARTICLE
}

\section{Nitrogen-Doped Sponge Ni Fibers as Highly Efficient Electrocatalysts for Oxygen Evolution Reaction}

Cite as

Nano-Micro Lett.

(2019) $11: 21$

Received: 26 January 2019

Accepted: 22 February 2019

Published online: 9 March 2019

(C) The Author(s) 2019

\author{
Kaili Zhang ${ }^{1}$, Xinhui Xia ${ }^{1,6}{ }^{凶}$, Shengjue Deng ${ }^{1}$, Yu Zhong ${ }^{1}$, Dong Xie ${ }^{2}$, Guoxiang Pan $^{3}$, \\ Jianbo Wu ${ }^{4}$, Qi Liu ${ }^{5}$, Xiuli Wang ${ }^{1}$, Jiangping $\mathrm{Tu}^{1} \bowtie$ \\ $\triangle$ Xinhui Xia, helloxxh@zju.edu.cn; Jiangping Tu, tujp@zju.edu.cn \\ 1 State Key Laboratory of Silicon Materials, Key Laboratory of Advanced Materials and Applications \\ for Batteries of Zhejiang Province, Department of Materials Science and Engineering, Zhejiang University, \\ Hangzhou 310027, People's Republic of China \\ 2 Guangdong Engineering and Technology Research Center for Advanced Nanomaterials, School \\ of Environment and Civil Engineering, Dongguan University of Technology, Dongguan 523808, \\ People's Republic of China \\ 3 Department of Materials Chemistry, Huzhou University, Huzhou 313000, People's Republic of China \\ 4 Zhejiang Provincial Key Laboratory for Cutting Tools, Taizhou University, Taizhou 318000, \\ People's Republic of China \\ 5 Department of Physics, City University of Hong Kong, Kowloon 999077, Hong Kong \\ 6 Key Laboratory of Advanced Energy Materials Chemistry (Ministry of Education), College of Chemistry, \\ Nankai University, Tianjin 300071, China
}

\section{HIGHLIGHTS}

- Freestanding N-doped sponge Ni micro/nanofibers exhibit a porous sponge structure.

- An N-doping strategy is adopted to optimize the catalytic activity.

- $\gamma$-NiOOH is identified as active phase by XPS and NEXAFS analyses.

\begin{abstract}
Controllable synthesis of highly active micro/nanostructured metal electrocatalysts for oxygen evolution reaction (OER) is a particularly significant and challenging target. Herein, we report a 3D porous sponge-like Ni material, prepared by a facile hydrothermal method and consisting of cross-linked micro/nanofibers, as an integrated binder-free OER electrocatalyst. To further enhance the electrocatalytic performance, an N-doping strategy is applied to obtain $\mathrm{N}$-doped sponge $\mathrm{Ni}(\mathrm{N}-\mathrm{SN})$ for the first time, via $\mathrm{NH}_{3}$ annealing. Due to the combination of the unique conductive sponge structure and $\mathrm{N}$ doping, the as-obtained

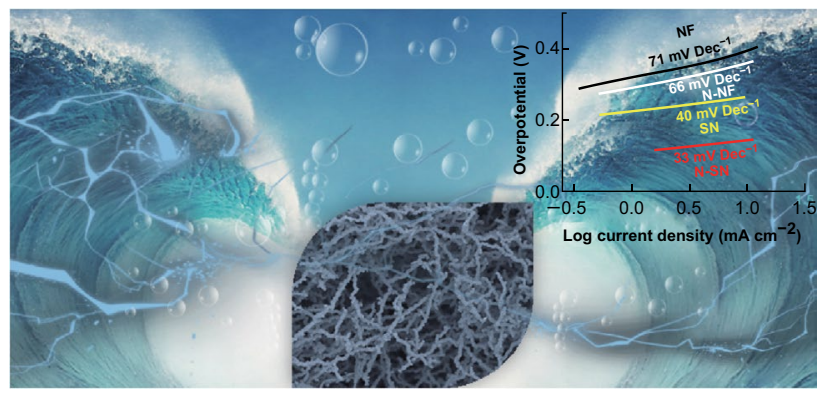
$\mathrm{N}-\mathrm{SN}$ material shows improved conductivity and a higher number of active sites, resulting in enhanced OER performance and excellent stability. Remarkably, N-SN exhibits a low overpotential of $365 \mathrm{mV}$ at $100 \mathrm{~mA} \mathrm{~cm}^{-2}$ and an extremely small Tafel slope of $33 \mathrm{mV}$ dec ${ }^{-1}$, as well as superior long-term stability, outperforming unmodified sponge Ni. Importantly, the combination of X-ray photoelectron spectroscopy and near-edge X-ray adsorption fine structure analyses shows that $\gamma$-NiOOH is the surface-active phase for OER. Therefore, the combination of conductive sponge structure and $\mathrm{N}$-doping modification opens a new avenue for fabricating new types of high-performance electrodes with application in electrochemical energy conversion devices.
\end{abstract}

KEYWORDS Oxygen evolution reaction; Electrocatalysis; Nickel; Sponge Structure; Electrochemical energy conversion 


\section{Introduction}

Owing to the huge concerns associated with serious environmental pollution and rapid fossil energy consumption, the development of clean and renewable energy technologies has become a vital task [1-4]. The oxygen evolution reaction (OER), as a key process in water splitting and rechargeable metal-air batteries, has attracted considerable attention for decades [5-14]. However, the OER is a kinetically sluggish process with a high overpotential, which calls for efficient electrocatalysts that can reduce the overpotential and improve the reaction efficiency [15-19]. Currently, noble metal oxides such as iridium/ruthenium oxides $\left(\mathrm{IrO}_{2} /\right.$ $\mathrm{RuO}_{2}$ ) set the benchmark for OER electrocatalysts [20-22]. However, the scarcity, prohibitive cost, and poor long-term durability of these materials restrict their wide application $[23,24]$. Therefore, it is highly desirable to develop highperformance and cost-effective OER catalysts.

In this context, transition metals are considered as potential alternatives and are attracting worldwide attention due to their reasonable cost, natural abundance, high conductivity, and outstanding stability [25-29]. Among these systems, nickel-based materials show a promising potential and have been extensively studied as efficient electrocatalysts for the water oxidation reaction. Nevertheless, nickel-based composites in bulk form are not competitive for electrocatalytic applications, owing to their low specific surface area and lack of exposed reactive sites. Hence, appropriate strategies, including compositing with conductive matrices or supports, rational structure design, and doping with heteroatoms, have been adopted in order to improve their electrocatalytic activity. For instance, $\mathrm{Xu}$ and coworkers designed nickel nanoparticles encapsulated in $\mathrm{N}$-doped graphene (denoted as $\mathrm{Ni} @ \mathrm{NC}$ ) by annealing a Ni-based metal-organic framework (MOF) and achieved an overpotential of $280 \mathrm{mV}$ at the current density of $10 \mathrm{~mA} \mathrm{~cm}{ }^{-2}$, with a small Tafel slope of $45 \mathrm{mV} \mathrm{dec}^{-1}$ [30]. Liu et al. [31] fabricated nickel nanoparticles encapsulated in $\mathrm{N}$-doped carbon nanotubes $(\mathrm{Ni} / \mathrm{N}-\mathrm{CNTs})$ exhibiting an overpotential of $590 \mathrm{mV}$ at $10 \mathrm{~mA} \mathrm{~cm}^{-2}$ and a Tafel slope of $138 \mathrm{mV} \mathrm{dec}^{-1}$, as well as high OER durability. Despite the enhanced electrochemical performance, the above materials still suffer from poor active site exposure and limited contact with electrolyte due to annealing-induced aggregation at high temperatures and structural collapse during rapid evolution of oxygen gas, resulting in significant performance degradation [32]. At variance with powders and substrate-assisted materials, selfsupported binder-free metal electrocatalysts can be directly used as electrodes with increased exposure of active sites and improved electrical conductivity; this avoids the use of binders and additives while enabling full utilization of the electrode-electrolyte interface, leading to remarkable catalytic performance. More recently, nano/microstructured sponge Ni with high electrical conductivity has emerged as a novel self-supported metal network, but no OER applications have been reported.

In the present work, we report for the first time $\mathrm{N}$-doped sponge nickel (denoted as N-SN), composed of interconnected Ni micro/nanofibers, as a binder-free high-efficiency OER catalysts; the N-SN material was prepared by a hydrothermal method followed by annealing in $\mathrm{NH}_{3}$ and exhibits a unique $3 \mathrm{D}$ porous structure and high electronic conductivity. The as-prepared N-SN micro/nanofibers have an open porous framework and consist of secondary nanosheets, which can significantly increase the surface area accessible to the electrolyte and expose a higher number of active sites. Due to the N-doping strategy and favorable conductive sponge architecture, the N-SN catalyst displays a remarkable OER performance, with a relatively low overpotential of $365 \mathrm{mV}$ (vs. reversible hydrogen electrode, RHE) at $100 \mathrm{~mA} \mathrm{~cm}^{-2}$, a Tafel slope of $33 \mathrm{mV} \mathrm{dec}^{-1}$, and high stability. Further analyses, including X-ray photoelectron spectroscopy (XPS) and near-edge X-ray adsorption fine structure (NEXAFS) tests, were used to investigate the electrocatalytic OER mechanism of N-SN. Our study can draw considerable attention on substrate-free metal materials as high-performance electrocatalysts.

\section{Experimental}

\subsection{Material Synthesis}

In a typical synthesis, $1 \mathrm{~g}$ nickel acetate $\left(\mathrm{Ni}(\mathrm{Ac})_{2}\right)$ was first dissolved in $100 \mathrm{~mL}$ deionized water and then, $10 \mathrm{~mL}$ hydrazine hydrate was added dropwise to the resulting aqueous solution. After stirring for $30 \mathrm{~min}$, the above solution was transferred into a Teflon-lined steel autoclave, which was kept at $150{ }^{\circ} \mathrm{C}$ for $12 \mathrm{~h}$. After naturally cooling and drying overnight, sponge $\mathrm{Ni}$ was obtained successfully. Then, the as-prepared sponge $\mathrm{Ni}$ was annealed at $300{ }^{\circ} \mathrm{C}$ for $2 \mathrm{~h}$ under 
$\mathrm{NH}_{3}(50 \mathrm{sccm})$ atmosphere to obtain N-SN. For comparison, $\mathrm{N}$-doped Ni foam (N-NF) was synthesized by following a similar procedure to that used to prepare $\mathrm{N}-\mathrm{SN}$, except that $\mathrm{Ni}$ foam (NF) was used as the skeleton. Commercial nickel foam $\left(1.0 \times 1.0 \mathrm{~cm}^{2}\right)$ was ultrasonically cleaned before use in hydrochloric acid $\left(1 \mathrm{~mol} \mathrm{~L}^{-1}\right)$, ethanol, and deionized water.

\subsection{Material Characterization}

The morphologies and microstructures of the samples were characterized by field-emission scanning electron microscopy (FESEM, SU8010) and high-resolution transmission electron microscopy (HRTEM, JEM 2100F). X-ray diffraction (XRD) patterns were collected on a Rigaku D/Max2550 instrument with $\mathrm{Cu} \mathrm{K}_{\alpha}$ radiation. XPS measurements were performed using an ESCALAB 250Xi spectrometer with an $\mathrm{Al} K_{\alpha}$ source. Brunauer-Emmett-Teller (BET) surface area distributions were obtained with a pore size analyzer (JW-BK112). Ni L-edge NEXAFS spectra were measured at the photoemission end station of beamline BL10B of the National Synchrotron Radiation Laboratory (NSRL) in Hefei, China. A bending magnet was connected to the beamline, equipped with three gratings covering photon energies from 100 to $1000 \mathrm{eV}$. In this experiment, the samples were kept in the total electron yield mode under an ultrahigh vacuum at $5 \times 10^{-10} \mathrm{mbar}$. The resolving power of the grating was typically $E / \Delta E=1000$, and the photon flux was $1 \times 10^{-10}$ photons $\mathrm{s}^{-1}$. Spectra were collected at energies from 831.4 to $884.6 \mathrm{eV}$ in $0.2 \mathrm{eV}$ energy steps.

\subsection{Electrochemical Measurements}

The OER performances of all samples were tested in a typical three-electrode configuration using an electrochemical workstation ( $\mathrm{CH}$ Instrument 660D). The synthesized samples $\left(1.0 \times 1.0 \mathrm{~cm}^{2}\right)$ were used as the working electrode, while a standard $\mathrm{Hg} / \mathrm{HgO}$ electrode and a Pt foil were used as the reference and counter electrode, respectively. The electrolyte was a $1 \mathrm{M} \mathrm{KOH}$ aqueous solution. Potentials were referenced to the RHE by adding $0.9254 \mathrm{~V}$. Twenty cyclic voltammetry (CV) cycles were performed to obtain a steady current. Then, linear sweep voltammetry (LSV) curves were obtained at the scan rate of $5 \mathrm{mV} \mathrm{s}^{-1}$ in the potential range from 0.2 to $1.2 \mathrm{~V}$ versus $\mathrm{Hg} / \mathrm{HgO}$ electrode. Tafel slopes were derived from the LSV curves. Moreover, electrochemical impedance spectroscopy (EIS) measurements were performed at the same polarization voltage for each sample, with a current density of around $10 \mathrm{~mA} \mathrm{~cm}^{-2}$ and within the frequency range from $0.01 \mathrm{~Hz}$ to $100 \mathrm{kHz}$. In order to evaluate the stability of the samples, long-term chronopotentiometry measurements were continuously conducted for $24 \mathrm{~h}$ at a constant current density of $10 \mathrm{~mA} \mathrm{~cm}{ }^{-2}$.

\section{Results and Discussion}

Figure 1 illustrates the morphology and element distribution of the as-prepared N-SN, obtained by the combination of hydrothermal synthesis and thermal $\mathrm{NH}_{3}$ treatment. As shown in Fig. 1a, b, the as-obtained N-SN is a soft and porous material with a freestanding structure and good mechanical stability. It can be randomly trimmed and directly used as a binder-free electrode for OER. During the hydrothermal process, nanoplates of the precursor nickel complex are formed first; then, they are directly reduced to nickel particles with the assistance of $\mathrm{OH}^{-}$ions in solution and hydrazine molecules in the nickel nanoplates. It is believed that the $\mathrm{Ni}^{2+}$ cations in solution are reduced on the surface of the particles, which are then joined end-toend to form sponge Ni. SEM images of N-SN are shown in Fig. 1c-f. The interconnected N-SN micro/nanofibers form a 3D porous architecture and leave large empty spaces, which are favorable for fast ion/electron transport. Interestingly, the N-SN micro/nanofibers show a hierarchical structure consisting of cross-linked nanosheets with thicknesses of $50-150 \mathrm{~nm}$. It should be noted that there are little differences in the morphologies of N-SN and sponge nickel (Fig. S1a). Both samples possess a hierarchical rough surface and porous structure. Compared with the NF and N-NF samples, possessing a smooth surface (Fig. S2a, b), the N-SN sample with smaller micro/nanofibers and rough nanosheet surfaces shows a larger surface area and could supply a higher number of active sites in the electrochemical reaction. Moreover, $\mathrm{N}$-doped nickel foam (which was also fabricated by $\mathrm{NH}_{3}$ treatment) shows almost the same smooth texture morphology as NF (Fig. S2b), revealing that the $\mathrm{NH}_{3}$ treatment does not alter the morphology. In addition, energy-dispersive spectroscopy (EDS) elemental mapping images (Fig. 1g) 

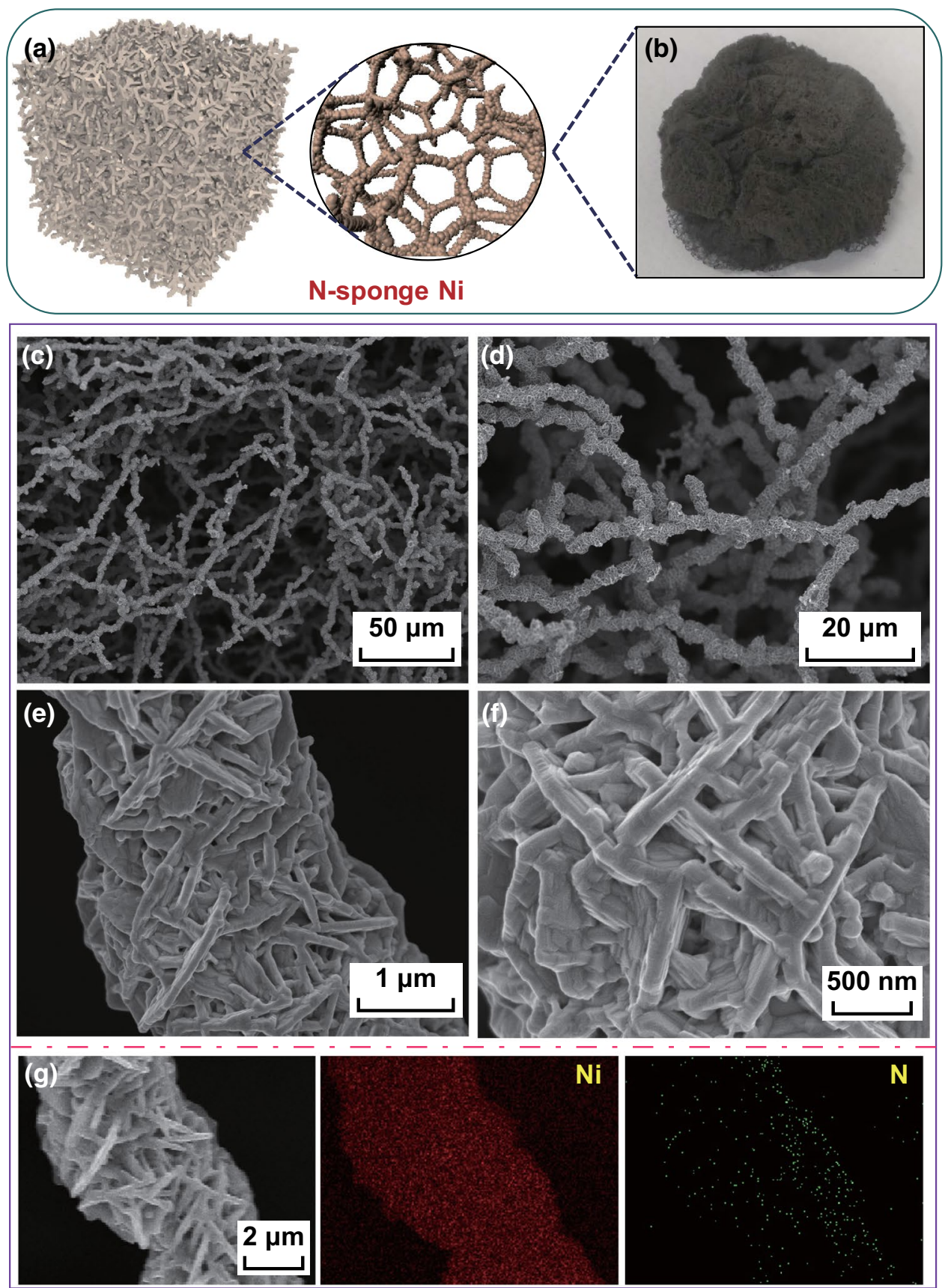

Fig. 1 a Schematic illustration of N-SN. b Optical image of N-SN. c-f SEM images of N-SN. $\mathbf{g}$ EDS elemental mapping images of Ni and N in $\mathrm{N}-\mathrm{SN}$

reveal the presence and uniform distribution of $\mathrm{Ni}$ and $\mathrm{N}$ in $\mathrm{N}-\mathrm{SN}$, confirming that $\mathrm{N}$ was successfully doped in the as-prepared sponge $\mathrm{Ni}$.

BET measurements were carried out to examine the porous nature and determine the surface area of the samples. The $\mathrm{N}_{2}$ adsorption/desorption isotherm curves are shown in
Fig. S3. The as-prepared N-SN electrode exhibits a specific surface area of $44.4 \mathrm{~m}^{2} \mathrm{~g}^{-1}$, much larger than that of SN $\left(23.7 \mathrm{~m}^{2} \mathrm{~g}^{-1}\right)$, N-NF $\left(13.6 \mathrm{~m}^{2} \mathrm{~g}^{-1}\right)$, and NF $\left(6.2 \mathrm{~m}^{2} \mathrm{~g}^{-1}\right)$, indicating that the $\mathrm{N}$ doping and sponge structure result in larger surface areas and are beneficial for exposing a higher 
number of active sites, resulting in improved utilization of the active materials.

Further insights into the microstructure of the N-SN arrays are provided by the transmission electron microscopy (TEM) analysis. Figure 2a presents a typical lowmagnification TEM image of a single $\mathrm{N}-\mathrm{SN}$ fiber, revealing a diameter of 1.5-2 $\mu \mathrm{m}$. Each fiber is composed of crosslinked nanosheets (Fig. 2b) as secondary building blocks (Fig. 2c). The HRTEM image of N-SN (Fig. 2d) shows a lattice fringe of $\sim 0.21 \mathrm{~nm}$, corresponding to the (111) planes of Ni (JCPDS No. 04-0850). Compared to the TEM image of SN (Fig. S1b), there are no distinct changes in the appearance of each N-SN fiber. The corresponding diffraction rings in the selected-area electron diffraction (SAED) pattern confirm the nickel phase (JCPDS No. 04-0850) of N-SN.

The crystallographic structure and composition of the integrated N-SN samples were further characterized by XRD and XPS (Fig. 3). All identified peaks at $44.5^{\circ}, 51.8^{\circ}$, and $76.4^{\circ}$ can be indexed to the cubic nickel phase (JCPDS No. 04-0850, Fig. 3a). Compared to nickel foam, peak broadening (with increased full width at half maximum, FWHM) is observed for sponge nickel. According to the Scherrer formula, the peak broadening can be attributed to a reduced particle size. In addition, XPS measurements were employed to investigate the elemental composition of N-SN. In the $\mathrm{Ni}$ spectra of N-SN (Fig. 3b), the peaks at 852.8 and $870.1 \mathrm{eV}$ are characteristic of Ni metal [33], while the peaks at binding energies at $855.8,853.7$, and $873.7 \mathrm{eV}$ are assigned to $\mathrm{Ni}^{2+}$, indicating the surface oxidation of N-SN. The other two satellite peaks at 860.8 and $879.1 \mathrm{eV}$ are in good agreement with those of the $\mathrm{Ni}^{2+}$ state [34]. In the $\mathrm{N} 1 \mathrm{~s}$ spectra, the peak at $397.8 \mathrm{eV}$ corresponds to $\mathrm{N}-\mathrm{Ni}$ bonds, while the $\mathrm{N} 1$ s peak shoulder at $399.4 \mathrm{eV}$ originates from the $\mathrm{N}-\mathrm{Ni}-\mathrm{O}$ bond (Fig. 3c) [35]. Moreover, the O 1s peaks (Fig. 3d) are attributed to the surface $\mathrm{NiO}$ resulting from surface oxidation of N-SN. The O 1s spectra consist of three main peaks and two weaker peaks at about 529.5 and $533.3 \mathrm{eV}$, which are attributed to lattice oxygen and adsorbed water, respectively. The most intense peak at $531.5 \mathrm{eV}$ is due to surface hydroxyl groups [34].

The electrochemical OER properties of the four samples were investigated and thoroughly compared. The OER activities of NF, N-NF, SN, and N-SN were examined through LSV measurements at a scan rate of $5 \mathrm{mV} \mathrm{s}^{-1}$ (Fig. 4a). In
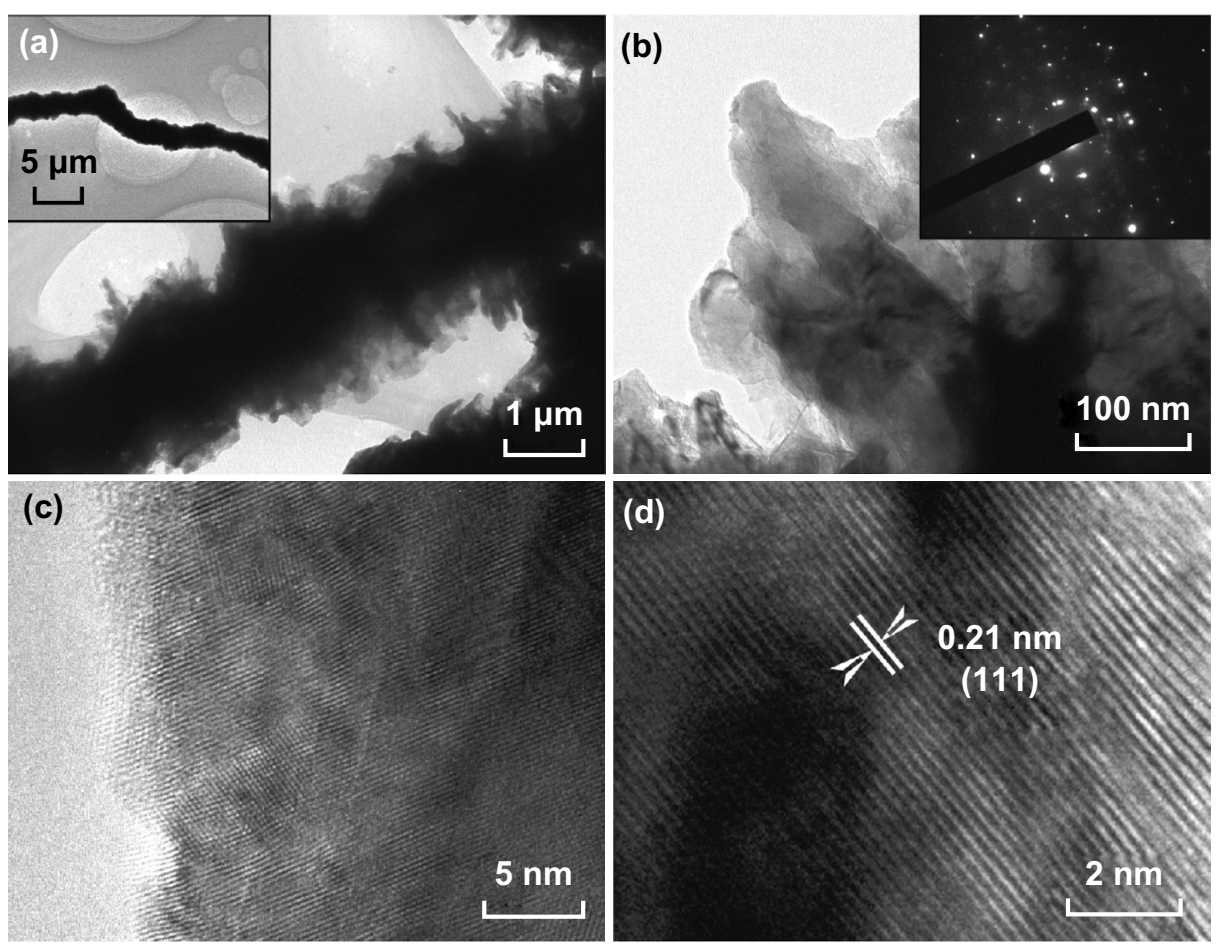

Fig. 2 a-d TEM and HRTEM images of N-SN. The inset images in (a, b) show a low-magnification TEM image and the SAED pattern 

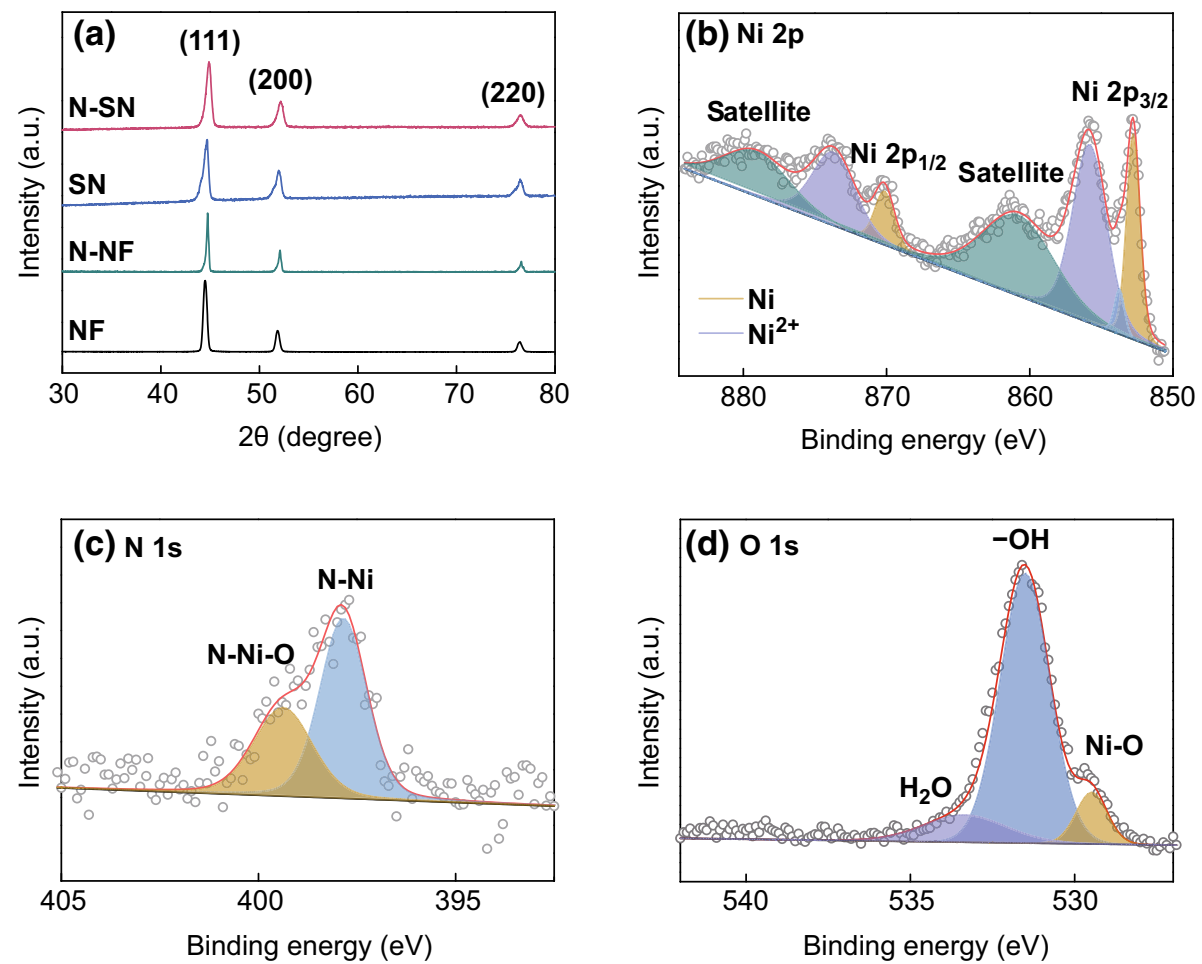

Fig. 3 a XRD patterns of NF, N-NF, SN, and N-SN. XPS spectra of initial N-SN before cycling: b Ni 2p, c N 1s, d O 1s

our case, the comparison of the overpotentials of the different samples at $10 \mathrm{~mA} \mathrm{~cm}^{-2}$ would not be accurate, due to the presence of strong redox peaks; hence, we selected the overpotentials at $100 \mathrm{~mA} \mathrm{~cm}^{-2}$ for the comparison. As shown in Fig. 4a, N-SN presents an extremely low overpotential of $365 \mathrm{mV}$ (vs. RHE) at a current density of $100 \mathrm{~mA} \mathrm{~cm}^{-2}$, lower than that of SN $(549 \mathrm{mV}), \mathrm{N}-\mathrm{NF}(565 \mathrm{mV})$, and $\mathrm{NF}$ $(645 \mathrm{mV})$. Clearly, $\mathrm{SN}$ exhibits better OER performance than NF, implying that the design of the micro/nanostructure helps obtaining a higher number of active sites and a faster ion/electron transfer path, which accelerate the water oxidation process. The OER performances of N-SN and N-NF exhibit a significant improvement compared to those of the unmodified SN and NF, respectively; this can be ascribed to the introduction of $\mathrm{N}$ heteroatoms, which improves the electronic conductivity $[1,36,37]$. The superior OER catalytic performance of $\mathrm{N}-\mathrm{SN}$ can be ascribed to the combination of its micro/nanoscale structure and enhanced electrical conductivity. The Tafel slopes in Fig. $4 \mathrm{~b}$ were used to investigate the OER kinetics. The Tafel equation $\eta=a+b \log j$ (where $\eta$, $j, b$, and $a$ represent the overpotential, current density, Tafel slope, and a constant depending on the electrode materials, respectively) can be applied in the high-polarization region. At low polarization values, the dependence of the current on the polarization is usually linear, rather than logarithmic. As a result, the current range between 1 and $10 \mathrm{~mA} \mathrm{~cm}{ }^{-2}$ is explored. A smaller Tafel slope denotes a faster OER kinetics [38], because of the faster increase in current densities with increasing overpotentials. The Tafel slope of SN is approximately $40 \mathrm{mV} \mathrm{dec}-1$, which further decreases to $33 \mathrm{mV} \mathrm{dec}^{-1}$ for $\mathrm{N}-\mathrm{SN}$, while the corresponding values of N-NF and NF reach 66 and $71 \mathrm{mV} \mathrm{dec}^{-1}$, respectively. The lowest Tafel slope of N-SN denotes its fastest kinetics [39-41], which was further confirmed by EIS measurements. Figure $4 \mathrm{c}$ shows the Nyquist plots of the four samples at the overpotential of $195 \mathrm{mV}$. In the high-frequency region, the $x$-axis intercept of N-SN is smaller than that of $\mathrm{SN}, \mathrm{N}-\mathrm{NF}$, and NF, suggesting that the micro/nanostructures of SN show a smaller resistance, and that $\mathrm{N}$ doping is beneficial for further enhancing the electronic conductivity. This reveals that the combination of micro/nanostructures and $\mathrm{N}$ doping in N-SN promotes faster electron transfer and ion diffusion, thus leading to better OER catalytic performances. 

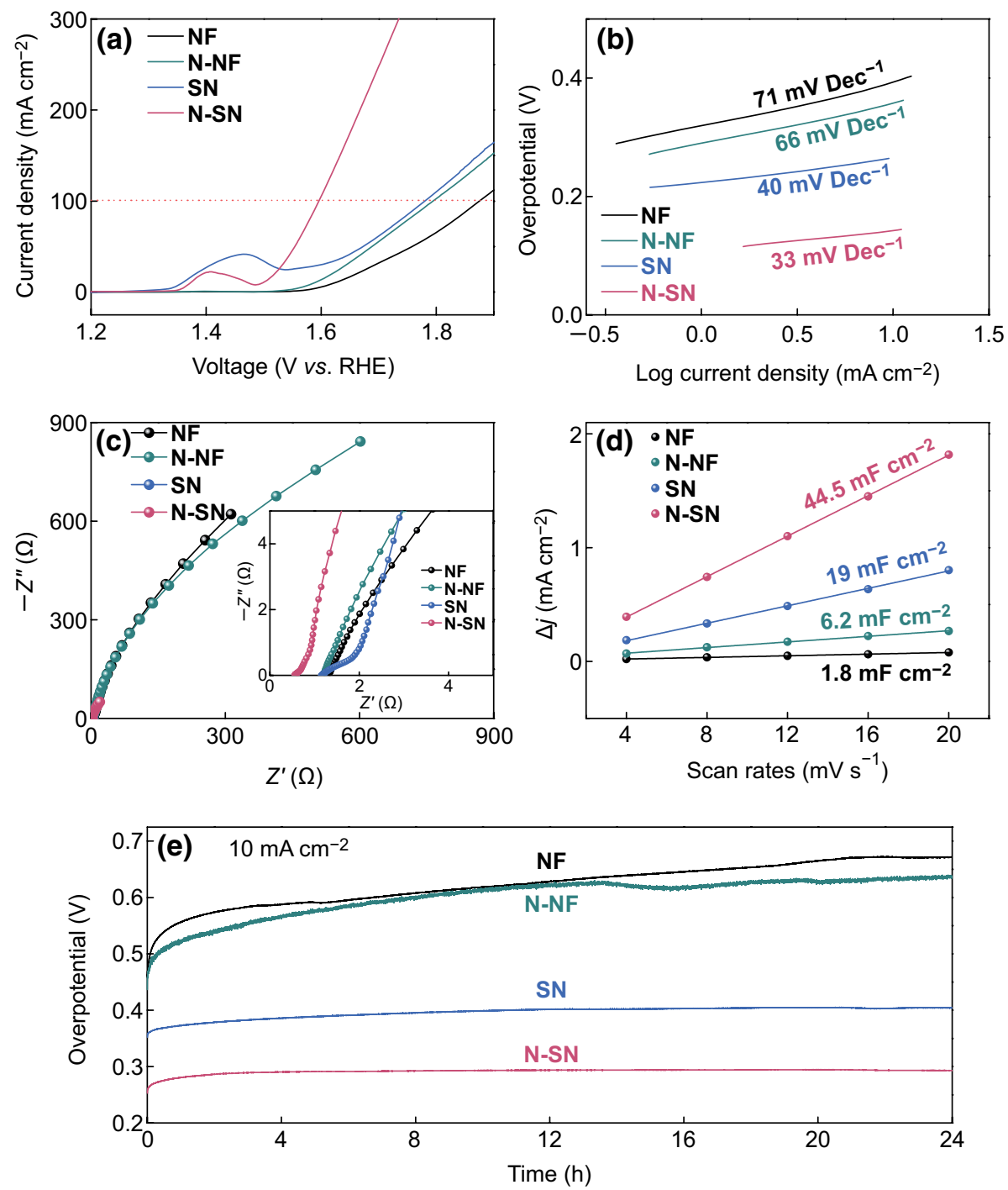

Fig. 4 OER performance characterization and comparison of NF, N-NF, SN, and N-SN. a LSV curves, b Tafel slopes, $\mathbf{c}$ Nyquist plots, $\mathbf{d}$ current densities obtained at different scan rates, e electrocatalytic stability at a current density of $10 \mathrm{~mA} \mathrm{~cm}-2$

The electrochemical active surface areas (ECSAs) of $\mathrm{N}-\mathrm{SN}, \mathrm{SN}, \mathrm{N}-\mathrm{NF}$, and NF were estimated from the electrochemical double-layer capacitance $\left(C_{\mathrm{dl}}\right)$ of each catalytic surface. The plots in Fig. 4d were obtained by measuring the non-Faradaic capacitive current associated with doublelayer charging from the scan rate dependence of the cyclic voltammograms (Fig. S4). The ECSA is expected to be linearly proportional to the $C_{\mathrm{dl}}$ value, which is equal to half the slope of the plot [42]. It should be pointed out that the $C_{\mathrm{dl}}$ value of N-SN is about $44.5 \mathrm{mF} \mathrm{cm}^{-2}$, about seven times higher than that of $\mathrm{N}-\mathrm{NF}\left(6.2 \mathrm{mF} \mathrm{cm}^{-2}\right)$, as well as higher than that of SN $\left(19 \mathrm{mF} \mathrm{cm}^{-2}\right)$ and $\mathrm{NF}\left(1.8 \mathrm{mF} \mathrm{cm}^{-2}\right)$. These obvious differences indicate that the tailored design and fabrication of $\mathrm{N}$-doped $\mathrm{SN}$ samples with micro/nanoscale structures lead to a marked increase in the number of active sites for OER, resulting in enhanced OER catalytic properties. On the other hand, the $C_{\mathrm{dl}}$ values of N-SN and N-NF are much higher than those of SN and NF, respectively, indicating that the $\mathrm{N}$-doping strategy also plays an important role in increasing the electrochemical active surface area. It can be thus be concluded that the combination of $\mathrm{N}$ doping and micro/nanostructure design takes full advantage of the available electrochemical active sites, improving the catalytic performance of the samples. The turnover frequency (TOF) is regarded as the best parameter to compare the intrinsic activities of electrocatalysts at various 
loadings. Assuming that all metal atoms in the samples are active and accessible to the electrolyte, the TOF values can be obtained according to the equation TOF $=i N_{\mathrm{A}} /\left(4 F N_{\text {atoms }}\right)$ where $i, N_{\mathrm{A}}, F$, and $N_{\text {atoms }}$ represent the current density at a specific overpotential, the Avogadro constant, the Faraday constant, and the number of atoms or active sites, respectively $[43,44]$. The TOF of N-SN at the overpotential of $400 \mathrm{mV}$ is calculated to be $1.190 \mathrm{~s}^{-1}$, which is much higher than that of SN $\left(0.290 \mathrm{~s}^{-1}\right)$, N-NF $\left(0.137 \mathrm{~s}^{-1}\right)$, and NF $\left(0.065 \mathrm{~s}^{-1}\right)$, indicating a better intrinsic activity of N-SN. In addition, the Faradaic efficiency was obtained by comparing the measured gas volume with the theoretical one. The Faradaic efficiency of N-SN is about $100 \%$, because of the good agreement between the experimental and calculated volumes of evolved $\mathrm{O}_{2}$ (Fig. S5).

To access and compare the OER stability of our four samples, long-term chronopotentiometry measurements were conducted at $10 \mathrm{~mA} \mathrm{~cm}{ }^{-2}$ for $24 \mathrm{~h}$ (Fig. 5e). The curve obtained for NF presents a clear increasing trend, demonstrating a higher overpotential and worse durability in the OER process. The curve corresponding to N-NF displays large fluctuations in the later stages of the reaction, suggesting unsatisfactory durability. In contrast, the curves obtained for $\mathrm{SN}$ and $\mathrm{N}-\mathrm{SN}$ remain flat and no noticeable overpotential increases are observed, indicating that $\mathrm{N}-\mathrm{SN}$ and $\mathrm{SN}$ retain a steady OER activity. In particular, the fluctuations in the N-SN curve remain in the range of $10 \mathrm{mV}$, which demonstrates the excellent stability of N-SN. The overpotential at $10 \mathrm{~mA} \mathrm{~cm}^{-2}$ of $\mathrm{N}-\mathrm{SN}$ is much smaller than that of SN, N-NF, and NF. The SEM images of N-SN after 100 cycles (Fig. S5) confirm that the interconnected structures are preserved well, without collapse or breakage. The outstanding mechanical stability of N-SN is beneficial for retaining a good electrochemical durability in alkaline aqueous solution.

To further illustrate the OER mechanism of N-SN, XPS and NEXAFS were used to examine the samples after 100 cycles and identify the actual active materials on the surface of N-SN (Fig. 5). According to the XPS analysis, the active peaks before and after the OER cycles change considerably, indicating that pure N-SN is not the active surface materials for OER and new products are produced
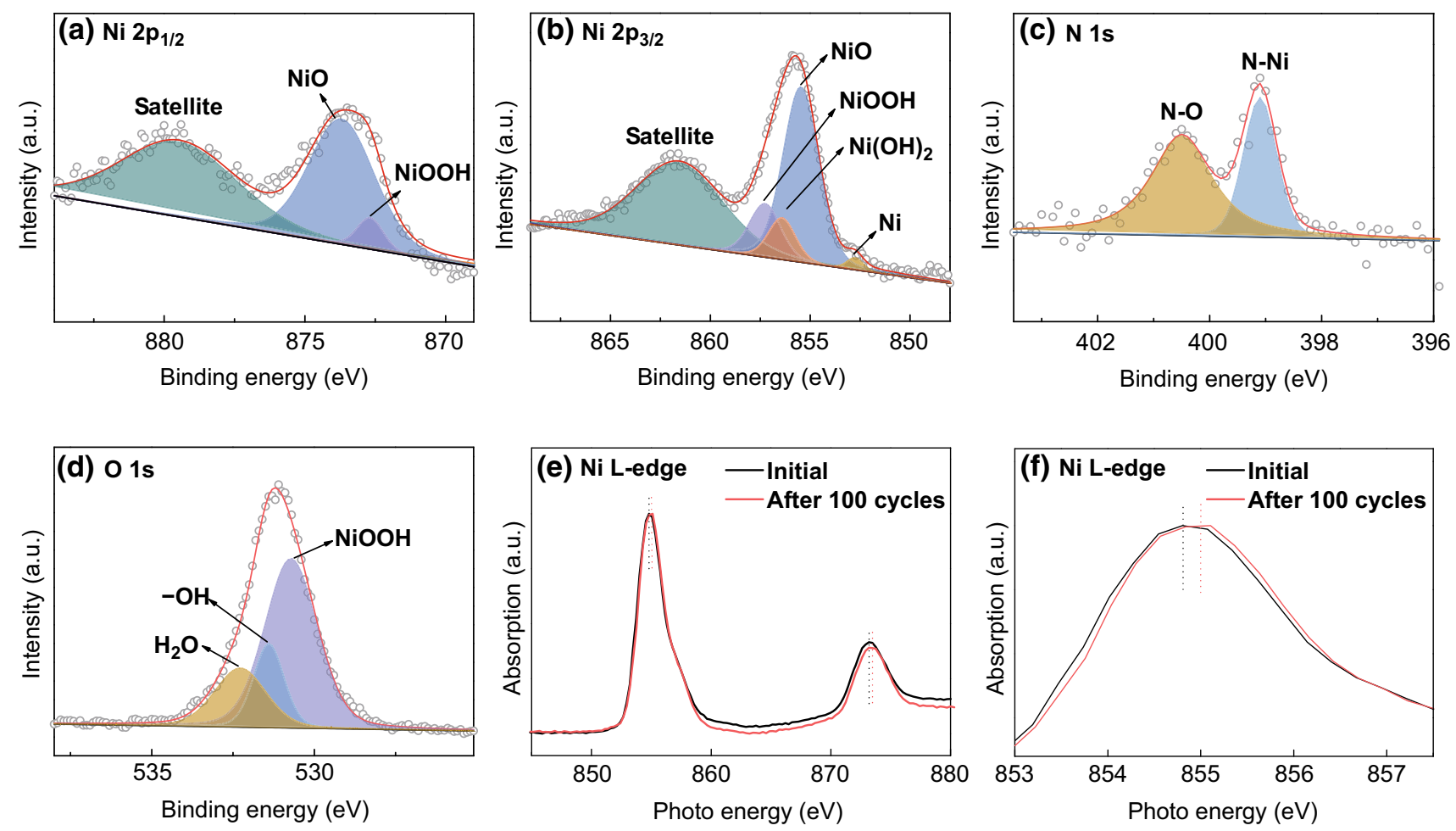

Fig. 5 XPS spectra of N-SN after 100 cycles. a Ni $2 p_{1 / 2}$, b Ni $2 p_{3 / 2}, \mathbf{c} N$ s, d O 1s. e-f NEXAFS spectra of N-SN before and after 100 OER cycles 
during the OER process. The Ni 2p spectra (Fig. 5a, b) show a marked decrease in the intensity of the peak at $852.8 \mathrm{eV}$, characteristic of $\mathrm{Ni}$ metal, while the previous peak at $870.1 \mathrm{eV}$ disappears. At the same time, the existence of $\mathrm{Ni}^{3+}$ species is evidenced by the peaks at 857.2 and $872.7 \mathrm{eV}$, assigned to $\gamma-\mathrm{NiOOH}$ originating from the surface oxidation of $\mathrm{N}-\mathrm{SN}$, which proceeds as follows: $\mathrm{Ni}^{2+}+3 \mathrm{OH}^{-}-3 \mathrm{e}^{-} \rightarrow \mathrm{NiOOH}+\mathrm{H}_{2} \mathrm{O}$ [45]. $\gamma-\mathrm{NiOOH}$ is probably the active species actually contributing to the OER process. The binding energy at $856.4 \mathrm{eV}$ corresponds to $\mathrm{Ni}(\mathrm{OH})_{2}$, because $\gamma-\mathrm{NiOOH}$ is not stable and will transform into $\mathrm{Ni}(\mathrm{OH})_{2}$ as follows: $\mathrm{NiOOH}+\mathrm{e}^{-}+\mathrm{H}_{2}$ $\mathrm{O} \rightarrow \mathrm{Ni}(\mathrm{OH})_{2}+\mathrm{OH}^{-}$[46]. Moreover, the peaks at 855.4 and $873.7 \mathrm{eV}$, attributed to $\mathrm{NiO}$, remain almost unchanged because of the surface oxidation of N-NS. Figure 5c shows peaks at 399.0 and $400.5 \mathrm{eV}$ corresponding to $\mathrm{N}-\mathrm{Ni}$ and $\mathrm{N}-\mathrm{O}$ bonds, respectively. The separation and shift of the $\mathrm{N}$ $1 \mathrm{~s}$ region reflect a change in chemical conditions after 100 cycles. A clear shift in the main $\mathrm{O} 1 \mathrm{~s}$ peaks is observed in Fig. 5d. The peak located at $530 \mathrm{eV}$ is characteristic of $\gamma-\mathrm{NiOOH}$, which is the typical species consistent with lattice oxygen [45]. This clearly demonstrates the existence of $\gamma-\mathrm{NiOOH}$, consistent with the Ni $2 \mathrm{p}$ spectra discussed above. The two remaining peaks at 531.4 and $532.3 \mathrm{eV}$ correspond to surface hydroxyl groups and adsorbed water, respectively. As shown in Fig. 5e, f, the L-edge NEXAFS results show the local structural variation around $\mathrm{Ni}$ sites in N-SN. The L-edge NEXAFS technique is one of the best tools to investigate the electronic structure of firstrow transition metals. The initial N-SN shows a sharp $\mathrm{L}_{3}$ maximum near $854.7 \mathrm{eV}$ and a relatively broad $\mathrm{L}_{2}$ edge, while a primary $\mathrm{L}_{3}$ peak near $855.0 \mathrm{eV}$ and a relatively similar $L_{2}$ region are observed after 100 cycles. Generally, the average $\mathrm{L}_{3}$ absorption centroid shifts to higher energies as $\mathrm{Ni}$ is oxidized from $\mathrm{Ni}^{\mathrm{I}}$ to $\mathrm{Ni}^{\mathrm{II}}, \mathrm{Ni}^{\mathrm{III}}$, and $\mathrm{Ni}^{\mathrm{IV}}$. In other words, the $\mathrm{L}_{3}$ peak of $\mathrm{N}-\mathrm{SN}$ shifts $\sim 0.3 \mathrm{eV}$ higher after 100 cycles, which indicates that $\mathrm{Ni}$ is oxidized to higher valence states during the OER process. In addition, as shown in the SEM images of N-SN after 100 cycles (Figs. S6, S7), the morphology of N-SN changes significantly and new small nanosheets are formed on the pristine secondary nanosheets due to the growth of $\gamma-\mathrm{NiOOH}$ and $\mathrm{Ni}(\mathrm{OH})_{2}$, in agreement with the XPS and NEXAFS analyses discussed above.

\section{Conclusion}

We have rationally designed and fabricated $\mathrm{N}$-doped sponge nickel as a novel and efficient OER electrocatalyst. Using a hydrothermal method combined with a thermal treatment in $\mathrm{NH}_{3}$ atmosphere, a self-supported N-SN consisting of micro/nanostructured fibers was successfully synthesized. The new material exhibits a 3D porous sponge skeleton with increased accessible surface area. The N-SN electrode shows high conductivity, large surface area, and abundant active sites, which result in excellent electrocatalytic performance, with low overpotential and high cycling stability. XPS and NEXAFS measurements were used to study the OER mechanism of $\mathrm{N}-\mathrm{SN} ; \boldsymbol{\gamma}-\mathrm{NiOOH}$, originating from the oxidation of $\mathrm{N}-\mathrm{SN}$ in alkaline solution, is identified as the actual active material for OER. In this work, we have not only demonstrated the potential of N-SN as a novel electrocatalyst, but also provided insights into how the sponge structure and $\mathrm{N}$-doping strategy can enhance the electrocatalytic performance.

Acknowledgements This work is supported by National Natural Science Foundation of China (Nos. 51728204 and 51772272), Fundamental Research Funds for the Central Universities (2018QNA4011), Qianjiang Talents Plan D (QJD1602029), and Startup Foundation for Hundred-Talent Program of Zhejiang University. The authors acknowledge the SEM/TEM support from Qiaohong He, Xiaokun Ding, and Fang Chen from Department of Chemistry, Zhejiang University.

Open Access This article is distributed under the terms of the Creative Commons Attribution 4.0 International License (http:// creativecommons.org/licenses/by/4.0/), which permits unrestricted use, distribution, and reproduction in any medium, provided you give appropriate credit to the original author(s) and the source, provide a link to the Creative Commons license, and indicate if changes were made.

Electronic supplementary material The online version of this article (https://doi.org/10.1007/s40820-019-0253-5) contains supplementary material, which is available to authorized users.

\section{References}

1. S. Deng, F. Yang, Q. Zhang, Y. Zhong, Y. Zeng et al., Phase modulation of (1T-2H)-MoSe 2 /TiC-C shell/core arrays via nitrogen doping for highly efficient hydrogen evolution reaction. Adv. Mater. 30, 1802223 (2018). https://doi.org/10.1002/ adma.201802223 
2. S. Deng, Y. Zhong, Y. Zeng, Y. Wang, Z. Yao et al., Directional construction of vertical nitrogen-doped 1T-2H MoSe 2 graphene shell/core nanoflake arrays for efficient hydrogen evolution reaction. Adv. Mater. 29(21), 1700748 (2017). https ://doi.org/10.1002/adma.201700748

3. F. Jiao, H. Frei, Nanostructured cobalt and manganese oxide clusters as efficient water oxidation catalysts. Energy Environ. Sci. 3(8), 1018-1027 (2010). https://doi.org/10.1039/c0020 $74 \mathrm{e}$

4. G. Fu, X. Yan, Y. Chen, L. Xu, D. Sun, J.M. Lee, Y. Tang, Boosting bifunctional oxygen electrocatalysis with 3D graphene aerogel-supported Ni/MnO particles. Adv. Mater. 30(5), 1704609 (2017). https://doi.org/10.1002/adma.201704609

5. S. Chen, J. Duan, M. Jaroniec, S.Z. Qiao, Nitrogen and oxygen dual-doped carbon hydrogel film as a substrate-free electrode for highly efficient oxygen evolution reaction. Adv. Mater. 26(18), 2925-2930 (2014). https://doi.org/10.1002/ adma.201305608

6. X. Li, Y. Fang, X. Lin, M. Tian, X. An, Y. Fu, R. Li, J. Jin, J. $\mathrm{Ma}$, MOF derived $\mathrm{Co}_{3} \mathrm{O}_{4}$ nanoparticles embedded in n-doped mesoporous carbon layer/MWCNT hybrids: extraordinary bifunctional electrocatalysts for OER and ORR. J. Mater. Chem. A 3(33), 17392-17402 (2015). https://doi.org/10.1039/c5ta0 $3900 \mathrm{~b}$

7. M. Shalom, D. Ressnig, X. Yang, G. Clavel, T.P. Fellinger, M. Antonietti, Nickel nitride as an efficient electrocatalyst for water splitting. J. Mater. Chem. A 3(15), 8171-8177 (2015). https://doi.org/10.1039/C5TA00078E

8. R. Souleymen, Z. Wang, C. Qiao, M. Naveed, C. Cao, Microwave-assisted synthesis of graphene-like cobalt sulfide freestanding sheets as an efficient bifunctional electrocatalyst for overall water splitting. J. Mater. Chem. A 6(2), 7592-7607 (2018). https://doi.org/10.1039/C8TA01266K

9. M.T.Y. Paul, B.B. Yee, D.R. Bruce, B.D. Gates, Hexagonal arrays of cylindrical nickel microstructures for improved oxygen evolution reaction. ACS Appl. Mater. Interfaces 9(8), 7036-7043 (2017). https://doi.org/10.1021/acsami.6b14129

10. H. Xu, J.X. Feng, Y. Tong, G.R. $\mathrm{Li}, \mathrm{Cu}_{2} \mathrm{O}-\mathrm{Cu}$ hybrid foams as high-performance electrocatalysts for oxygen evolution reaction in alkaline media. ACS Catalysis 7(2), 986-991 (2016). https://doi.org/10.1021/acscatal.6b02911

11. P. Zhang, L. Li, D. Nordlund, H. Chen, L. Fan, B. Zhang, X. Sheng, Q. Daniel, L. Sun, Dendritic core-shell nickel-ironcopper metal/metal oxide electrode for efficient electrocatalytic water oxidation. Nat. Commun. 9(1), 381 (2018). https ://doi.org/10.1038/s41467-017-02429-9

12. J. Wang, W. Zhang, Z. Zheng, J. Liu, C. Yu, Y. Chen, K. Ma, Dendritic core-shell Ni@Ni(Fe)OOH metal/metal oxyhydroxide electrode for efficient oxygen evolution reaction. Appl. Surf. Sci. 469, 731-738 (2019). https://doi.org/10.1016/j.apsus c. 2018.10 .232

13. D. Chen, M. Qiao, Y.-R. Lu, L. Hao, D. Liu, C.-L. Dong, Y. Li, S. Wang, Preferential cation vacancies in perovskite hydroxide for the oxygen evolution reaction. Angew. Chem. Int. Ed. 57(28), 8691-8696 (2018). https://doi.org/10.1002/ anie. 201805520
14. K. Gao, B. Wang, L. Tao, B.V. Cunning, Z. Zhang, S. Wang, R.S. Ruoff, L. Qu, Efficient metal-free electrocatalysts from $\mathrm{N}$-doped carbon nanomaterials: mono-doping and Co-doping. Adv. Mater. 1805121 (2018). https://doi.org/10.1002/ adma.201805121

15. K. Xu, P. Chen, X. Li, Y. Tong, H. Ding et al., Metallic nickel nitride nanosheets realizing enhanced electrochemical water oxidation. J. Am. Chem. Soc. 137(12), 4119-4125 (2015). https://doi.org/10.1021/ja5119495

16. W.T. Hong, M. Risch, K.A. Stoerzinger, A. Grimaud, S. Jin, S.H. Yang, Toward the rational design of non-precious transition metal oxides for oxygen electrocatalysis. Energy Environ. Sci. 8(5), 1404-1427 (2015). https://doi.org/10.1039/C4EE0 3869J

17. S. Mao, Z. Wen, T. Huang, Y. Hou, J. Chen, High-performance bi-functional electrocatalysts of 3D crumpled graphene-cobalt oxide nanohybrids for oxygen reduction and evolution reactions. Energy Environ. Sci. 7(2), 609-616 (2014). https://doi. org/10.1039/C3EE42696C

18. J. Ren, Z. Hu, C. Chen, Y. Yuan, Integrated Ni2p nanosheet arrays on three-dimensional $\mathrm{Ni}$ foam for highly efficient water reduction and oxidation. J. Energy Chem. 26(6), 1196-1202 (2017). https://doi.org/10.1016/j.jechem.2017.07.016

19. H. Liu, K. Hu, D. Yan, R. Chen, Y. Zou, H. Liu, S. Wang, Recent advances on black phosphorus for energy storage, catalysis, and sensor applications. Adv. Mater. 30(32), 1800295 (2018). https://doi.org/10.1002/adma.201800295

20. Y. Gong, Z. Xu, H. Pan, Y. Lin, Z. Yang, J. Wang, A 3D well-matched electrode pair of $\mathrm{Ni}-\mathrm{Co}-\mathrm{S} / \mathrm{Ni}-\mathrm{Co}-\mathrm{P}$ nanoarrays grown on nickel foam as a high-performance electrocatalyst for water splitting. J. Mater. Chem. A 6(26), 12506-12514 (2018). https://doi.org/10.1039/c8ta03163k

21. W. Zhou, X.J. Wu, X. Cao, X. Huang, C. Tan, J. Tian, H. Liu, J. Wang, H. Zhang, $\mathrm{Ni}_{3} \mathrm{~S}_{2}$ nanorods/Ni foam composite electrode with low overpotential for electrocatalytic oxygen evolution. Energy Environ. Sci. 6(10), 2921-2924 (2013). https:// doi.org/10.1039/C3EE41572D

22. J. Wang, H.X. Zhong, Y.L. Qin, X.B. Zhang, An efficient three-dimensional oxygen evolution electrode. Angew. Chem. Int. Ed. 52(20), 5248-5253 (2013). https://doi.org/10.1002/ anie. 201301066

23. K. Zhang, X. Xia, S. Deng, D. Xie, Y. Lu, Y. Wang, J. Wu, X. Wang, J. Tu, N-doped CoO nanowire arrays as efficient electrocatalysts for oxygen evolution reaction. J. Energy Chem. 37, 13-17 (2019). https://doi.org/10.1016/j.jechem.2018.11.013

24. S. Chen, J. Duan, J. Ran, M. Jaroniec, S.Z. Qiao, N-doped graphene film-confined nickel nanoparticles as a highly efficient three-dimensional oxygen evolution electrocatalyst. Energy Environ. Sci. 6(12), 3693-3699 (2013). https://doi. org/10.1039/c3ee42383b

25. J. Jiang, F. Sun, S. Zhou, W. Hu, H. Zhang et al., Atomic-level insight into super-efficient electrocatalytic oxygen evolution on iron and vanadium co-doped nickel (OXY) hydroxide. Nat. Commun. 9(1), 2885 (2018). https://doi.org/10.1038/s4146 7-018-05341-y 
26. L. Trotochaud, S.L. Young, J.K. Ranney, S.W. Boettcher, Nickel-iron oxyhydroxide oxygen-evolution electrocatalysts: the role of intentional and incidental iron incorporation. J. Am. Chem. Soc. 136(18), 6744-6753 (2014). https://doi. org/10.1021/ja502379c

27. M. Gao, W. Sheng, Z. Zhuang, Q. Fang, S. Gu, J. Jiang, Y. Yan, Efficient water oxidation using nanostructured $\alpha$-nickelhydroxide as an electrocatalyst. J. Am. Chem. Soc. 136(19), 7077-7084 (2014). https://doi.org/10.1021/ja502128j

28. X. Yang, J. Chen, Y. Chen, P. Feng, H. Lai, J. Li, X. Luo, Novel $\mathrm{Co}_{3} \mathrm{O}_{4}$ nanoparticles/nitrogen-doped carbon composites with extraordinary catalytic activity for oxygen evolution reaction (OER). Nano-Micro Lett. 10(1), 15 (2017). https:// doi.org/10.1007/s40820-017-0170-4

29. J. Zhang, X. Bai, T. Wang, W. Xiao, P. Xi, J. Wang, D. Gao, J. Wang, Bimetallic nickel cobalt sulfide as efficient electrocatalyst for Zn-air battery and water splitting. Nano-Micro Lett. 11(1), 2 (2019). https://doi.org/10.1007/s40820-018-0232-2

30. Y. Xu, W. Tu, B. Zhang, S. Yin, Y. Huang, M. Kraft, R. Xu, Nickel nanoparticles encapsulated in few-layer nitrogen-doped graphene derived from metal-organic frameworks as efficient bifunctional electrocatalysts for overall water splitting. Adv. Mater. 29(11), 1605957 (2017). https://doi.org/10.1002/ adma.201605957

31. Y. Liu, H. Jiang, Y. Zhu, X. Yang, C. Li, Transition metals ( $\mathrm{Fe} \mathrm{Co}$, and $\mathrm{Ni}$ ) encapsulated in nitrogen-doped carbon nanotubes as bi-functional catalysts for oxygen electrode reactions. J. Mater. Chem. A 4(5), 1694-1701 (2016). https ://doi.org/10.1039/c5ta10551j

32. G. Cai, W. Zhang, L. Jiao, S.-H. Yu, H.-L. Jiang, Templatedirected growth of well-aligned mof arrays and derived selfsupporting electrodes for water splitting. Chem 2(6), 791802 (2017). https://doi.org/10.1016/j.chempr.2017.04.016

33. P. Prieto, V. Nistor, K. Nouneh, M. Oyama, M. Abd-Lefdil, R. Díaz, XPS study of silver, nickel and bimetallic silvernickel nanoparticles prepared by seed-mediated growth. Appl. Surf. Sci. 258(22), 8807-8813 (2012). https://doi. org/10.1016/j.apsusc.2012.05.095

34. M.A. Peck, M.A. Langell, Comparison of nanoscaled and bulk NiO structural and environmental characteristics by XRD, XAFS, and XPS. Chem. Mater. 24(23), 4483-4490 (2012). https://doi.org/10.1021/cm300739y

35. N. Jiang, H.J. Zhang, S.N. Bao, Y.G. Shen, Z.F. Zhou, XPS study for reactively sputtered titanium nitride thin films deposited under different substrate bias. Physica B: Condens. Matter 352(1-4), 118-126 (2004). https://doi. org/10.1016/j.physb.2004.07.001

36. X. Ren, Q. Ma, P. Ren, Y. Wang, Synthesis of nitrogen-doped $\mathrm{MoSe}_{2}$ nanosheets with enhanced electrocatalytic activity for hydrogen evolution reaction. Int. J. Hydrog. Energy 43(32), 15275-15280 (2018). https://doi.org/10.1016/j.ijhyd ene.2018.06.122

37. J. Wang, T. Qiu, X. Chen, Y. Lu, W. Yang, N-doped carbon@ $\mathrm{Ni}-\mathrm{Al}_{2} \mathrm{O}_{3}$ nanosheet array@ graphene oxide composite as an electrocatalyst for hydrogen evolution reaction in alkaline medium. J. Power Sources 293, 178-186 (2015). https ://doi.org/10.1016/j.jpowsour.2015.05.080

38. S. Deng, Y. Zhong, Y. Zeng, Y. Wang, X. Wang, X. Lu, X. Xia, J. Tu, Hollow $\mathrm{TiO}_{2} @ \mathrm{Co}_{9} \mathrm{~S}_{8}$ core-branch arrays as bifunctional electrocatalysts for efficient oxygen/hydrogen production. Adv. Sci. 5(3), 1700772 (2017). https://doi. org/10.1002/advs.201700772

39. Z. Yao, X. Xia, D. Xie, Y. Wang, C.-A. Zhou, S. Liu, S. Deng, X. Wang, J. Tu, Enhancing ultrafast lithium ion storage of $\mathrm{Li}_{4} \mathrm{Ti}_{5} \mathrm{O}_{12}$ by tailored $\mathrm{TiC} / \mathrm{C}$ core/shell skeleton plus nitrogen doping. Adv. Funct. Mater. 28(31), 1802756 (2018). https://doi.org/10.1002/adfm.201802756

40. X.H. Xia, S. Deng, X. Dong, Y. Wang, S. Feng, J.B. Wu, J. Tu, Boosting sodium ion storage by anchoring $\mathrm{MoO}_{2}$ on vertical graphene arrays. J. Mater. Chem. A 6(32), 1554615552 (2018). https://doi.org/10.1039/C8TA06232C

41. X.H. Xia, S. Deng, S. Feng, J.B. Wu, J. Tu et al., Hierarchical porous $\mathrm{Ti}_{2} \mathrm{Nb}_{10} \mathrm{O}_{29}$ nanospheres as superior anode materials for lithium ion storage. J. Mater. Chem. A 5(40), 21134-21139 (2017). https://doi.org/10.1039/C7TA07229E

42. C.C. Mccrory, S. Jung, J.C. Peters, T.F. Jaramillo, Benchmarking heterogeneous electrocatalysts for the oxygen evolution reaction. J. Am. Chem. Soc. 135(45), 16977-16987 (2013). https://doi.org/10.1021/ja407115p

43. Y. Xue, B. Huang, Y. Yi, Y. Guo, Z. Zuo, Y. Li, Z. Jia, H. Liu, Y. Li, Anchoring zero valence single atoms of nickel and iron on graphdiyne for hydrogen evolution. Nat. Commun. 9(1), 1460 (2018). https://doi.org/10.1038/s4146 7-018-03896-4

44. I.J. Godwin, M.E.G. Lyons, Enhanced oxygen evolution at hydrous nickel oxide electrodes via electrochemical ageing in alkaline solution. Electrochem. Commun. 32, 39-42 (2013). https://doi.org/10.1016/j.elecom.2013.03.040

45. A.N. Mansour, C.A. Melendres, Characterization of electrochemically prepared $\gamma$-NiOOH by XPS. Surface Sci. Spectra 3(3), 271-278 (1994). https://doi.org/10.1116/1.1247756

46. T. Reier, Z. Pawolek, S. Cherevko, M. Bruns, T. Jones et al., Molecular insight in structure and activity of highly efficient, low-Ir Ir-Ni oxide catalysts for electrochemical water splitting (OER). J. Am. Chem. Soc. 137(40), 13031-13040 (2015). https://doi.org/10.1021/jacs.5b07788 